\title{
Vibrational modes in nanocrystalline iron under high pressure
}

\author{
Alexander B. Papandrew, Alan F. Yue, and Brent Fultz \\ Division of Engineering and Applied Science, 138-78, California Institute of Technology, Pasadena, California 91125, USA \\ Itzhak Halevy \\ Physics Department, Nuclear Research Center-Negev, 84190 Beer-Sheva, Israel \\ Wolfgang Sturhahn, Thomas S. Toellner, and E. Ercan Alp \\ Advanced Photon Source, Argonne National Laboratory, Argonne, Illinois 60439, USA \\ Ho-kwang Mao \\ Geophysical Laboratory, Carnegie Institute of Washington, 5251 Broad Branch Road N.W., Washington, DC 20015, USA
}

(Received 7 November 2003; published 12 April 2004)

\begin{abstract}
The phonon density of states (DOS) of nanocrystalline ${ }^{57} \mathrm{Fe}$ was measured using nuclear resonant inelastic $\mathrm{x}$-ray scattering (NRIXS) at pressures up to $28 \mathrm{GPa}$ in a diamond anvil cell. The nanocrystalline material exhibited an enhancement in its DOS at low energies by a factor of 2.2. This enhancement persisted throughout the entire pressure range, although it was reduced to about 1.7 after decompression. The low-energy regions of the spectra were fitted to the function $A E^{n}$, giving values of $n$ close to 2 for both the bulk control sample and the nanocrystalline material, indicative of nearly three-dimensional vibrational dynamics. At higher energies, the van Hove singularities observed in both samples were coincident in energy and remained so at all pressures, indicating that the forces conjugate to the normal coordinates of the nanocrystalline materials are similar to the interatomic potentials of bulk crystals.
\end{abstract}

DOI: 10.1103/PhysRevB.69.144301

\section{INTRODUCTION}

In the last decade, neutron and x-ray inelastic scattering experiments have revealed significant distortions in the phonon density of states (DOS) of nanocrystalline metals and alloys compared to their coarse-grained counterparts. ${ }^{1-7}$ Notable among these differences is the presence of an increased number of vibrational modes at the extremes of the vibrational spectrum. Enhanced intensity above the high-energy cutoff of the bulk material has been attributed to phonon lifetime broadening caused by phonon interactions with grain boundaries $^{4-6}$ and recent measurements on iron have isolated a contribution from surface oxides. ${ }^{7}$

The low-energy region of the vibrational spectrum of nanocrystals is less well understood. There is general agreement that nanostructured materials have dynamical degrees of freedom that do not exist in bulk crystals, and these degrees of freedom make a significant contribution to their vibrational spectra at thermal energies. The physical origin of these vibrational modes has been the subject of speculation and debate. Cooperative dynamics of the crystals themselves were suggested, ${ }^{5,8}$ as were surface modes involving elastic discontinuities between nanocrystals. It has been suggested that nanocrystals could have novel two-dimensional vibrational modes owing to their large surface area. Some evidence for two-dimensional behavior has been provided by theory ${ }^{9,10}$ and experiment, ${ }^{11}$ although most experimental work reports no linear component of the phonon DOS at low energies. ${ }^{1,3-7}$ Nonintegral spatial dimensions of the lowfrequency modes have been suggested, ${ }^{12,13}$ such as fractal modes or "fractons." 14

If the low-frequency modes of nanostructures are associated with generalized forces that differ from those between
PACS number(s): 63.22. $+\mathrm{m}, 62.50 .+\mathrm{p}$, 81.07.Bc

atoms in crystals, their frequencies may respond differently to pressure. The present investigation was designed to test to what extent this is true, and to test for evidence of lowerdimensional dynamics. In addition, the compressions accessible in the diamond anvil cell are expected to induce mesoscopic changes such as the closing of pores and voids, eliminating their effect on the DOS. Samples of nanocrystalline and coarse-grained ${ }^{57} \mathrm{Fe}$ were compressed in a diamond anvil cell for measurements of nuclear resonant inelastic $\mathrm{x}$-ray scattering (NRIXS). The NRIXS technique provides direct access to the phonon DOS without the need for a lattice dynamics model, permitting analysis of the populations of low-frequency modes irrespective of their origin. ${ }^{15}$

\section{EXPERIMENT}

Nanocrystalline ${ }^{57} \mathrm{Fe}$ was prepared by the inert gasconsolidation technique. ${ }^{16}$ Approximately $30 \mathrm{mg}$ of iron enriched to $95 \%$ in the ${ }^{57} \mathrm{Fe}$ isotope was evaporated by resistive heating in a gas stream of $\mathrm{N}_{2}$ containing $10 \mathrm{vol} \% \mathrm{H}_{2}$ at a pressure of 2 Torr. Iron particles entrained in the gas flow impacted against a glass slide to form a thin film of ${ }^{57} \mathrm{Fe}$ of approximately $3 \mathrm{mg}$. The sample chamber was then sealed and transferred to an argon atmosphere where the iron film was coated with a thin layer of silicone oil to retard oxidation.

$\mathrm{X}$-ray-diffraction patterns were measured using Co $\mathrm{K} \alpha$ radiation to determine the crystal structure, grain size, and degree of oxidation of the nanocrystalline samples. Scanning and transmission electron microscopy were also performed on natural iron films deposited under identical conditions to establish morphology and to verify grain size. Representative transmission electron microscopy (TEM) micrographs of 
nanocrystalline ${ }^{57} \mathrm{Fe}$ are shown in Fig. 4.

NRIXS was performed at the synchrotron beamline 3-ID of the Advanced Photon Source (APS). The X-ray beam from the undulators was monochromated to a final resolution of 1 meV full width at half maximum (FWHM) by a silicon (4 0 0) (10 6 4) monochromator ${ }^{17}$ after initial bandpass filtering by a water-cooled (111) diamond. The lineshape delivered by the monochromator was measured by an avalanche photodiode mounted in the forward beam. The tails of this instrument resolution function were negligible at values of $\pm 5 \mathrm{meV}$.

Samples of nanocrystalline and polycrystalline bulk ${ }^{57} \mathrm{Fe}$ were loaded into separate piston-cylinder-type diamond anvil cells (DAC) optimized for use in NRIXS experiments. The specific design of these cells and the experimental configuration have been discussed elsewhere. ${ }^{18}$ Diamonds with $500 \mu \mathrm{m}$ culets were used, and silicone oil (Dow Corning DC-705) was employed as a pressure medium. Incoherent inelastic scattering spectra were collected by tuning the incident energy of the x-ray beam from the silicon monochromator in $0.25 \mathrm{meV}$ steps and counting the delayed photons from nuclear deexcitations. Spectra were collected in a range of $\pm 80 \mathrm{meV}$ around the Mössbauer resonance at a rate of 1 $\mathrm{h}$ per scan. Typically 10-12 scans were performed and added together to yield a total incoherent scattering function $S(E)$. The procedure reviewed by $\mathrm{Hu}$ et $a l .{ }^{19}$ was then applied to obtain the phonon DOS of both samples at each pressure. The pressure on the sample was measured before and after each set of scans by the ruby fluorescence technique ${ }^{20}$ to confirm pressure stability during the measurement. Nuclear forward scattering (NFS) spectra $^{21}$ were also measured at each pressure to monitor the crystalline phase of the sample, since the sensitivity of NFS to magnetic order allowed discrimination of the ferromagnetic bcc $\alpha$ phase from the nonmagnetic hcp $\epsilon$ phase. $^{22}$

\section{RESULTS}

X-ray-diffraction patterns of the ballistically consolidated films showed a bcc Fe structure with a small amount of oxide. The Scherrer equation for diffraction line broadening yielded an average particle size of $9 \mathrm{~nm}$. TEM performed with a Philips EM420 instrument operated at $120 \mathrm{kV}$ showed distinct individual crystallites, which can also be seen in Fig. 4. The average grain size seen in dark-field images was consistent with the x-ray results. Films examined with scanning electron microscopy (SEM) possessed regular, mildly rough surfaces and a uniform morphology through their entire thickness. Stylus profilometry and SEM measured an average thickness of $20 \mu \mathrm{m}$. Overall, the films were very similar to those described in our earlier report on NRIXS of nanocrystalline iron at ambient conditions. ${ }^{4}$

The phonon densities of states of both nanocrystalline and coarse-grained ${ }^{57} \mathrm{Fe}$ are presented in Fig. 1. All have been normalized to unity. The excess intensity in the nanocrystalline DOS below $20 \mathrm{meV}$ is obvious, but quantifying this excess intensity is challenging. Many authors adopt an arbitrary standard; we sought a systematic procedure for identifying this region. At long wavelengths, a three-dimensional

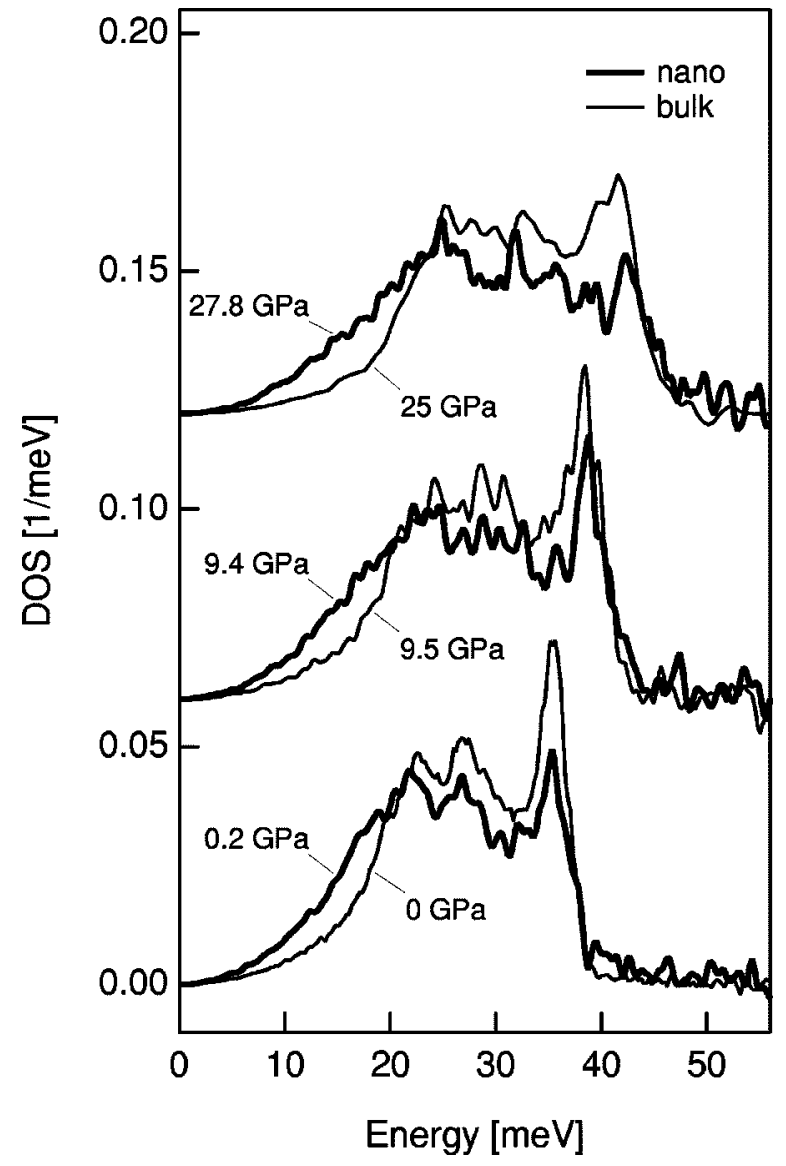

FIG. 1. Phonon density of states (DOS) of nanocrystalline and bulk ${ }^{57} \mathrm{Fe}$ at various pressures. The upper traces have been shifted vertically by 0.06 and $0.12 \mathrm{meV}^{-1}$ with respect to the data at ambient pressure.

solid has a parabolic DOS of the form $B E^{2}$, following the work of Debye. This parabola was assumed as a reasonable approximation of the "low-energy" part of the bulk iron DOS measured at ambient pressure. It was divided by the thermal correction factor $E[1-\exp (-E / k T)]$ and then fit to the inelastic scattering spectrum $S(E)$ of the bulk sample at ambient pressure, using $B$ as the only adjustable parameter. The limits of the fit were adjusted symmetrically until subsequent $\chi^{2}$ values for the fit approached a constant value. This was taken to delimit the range of applicability of the Debye model for bulk iron, and consequently the appropriate range for discussion. The limit obtained was $12 \mathrm{meV}$. The results of this fit are shown in Fig. 2. (Note that the calculated curve is close to a straight line. Fitting a straight line to a range of experimental data helps minimize problems with counting statistics.) By integrating the DOS curves, we were able to determine the fraction of the modes present in the samples with energies less than $12 \mathrm{meV}$. The fractions of low-energy modes at each pressure are presented in Fig. 3.

As the pressure on the sample is increased, the entire DOS shifts towards higher energies. This can be seen most easily in the movement of the longitudinal peak near the high-energy cutoff of the DOS. The pressure-induced phase transition of iron from the bcc to the hcp structure occurs in the region between 9 and $28 \mathrm{GPa}$, and nuclear forward scat- 


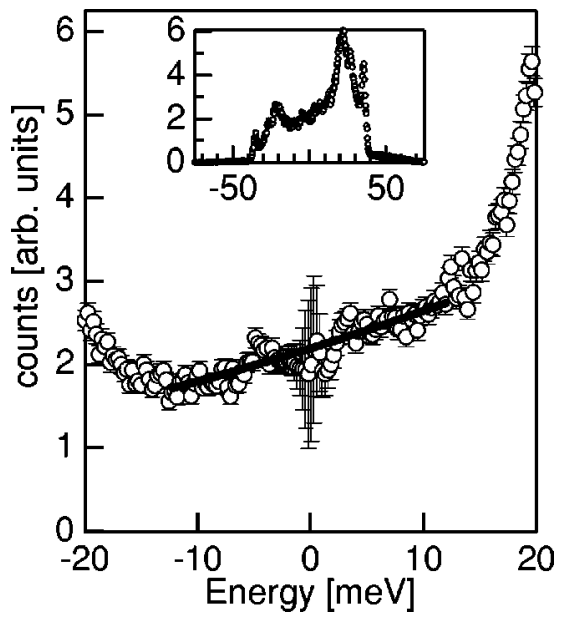

FIG. 2. Scattering data from bulk ${ }^{57} \mathrm{Fe}$ with elastic peak removed. The function $D(E)=B E /[1-\exp (-E / k T)]$ has been fit to the data in the interval $\pm 12 \mathrm{meV}$. The fit is shown as the black curve. The full energy range is shown inset.

tering NFS spectra confirmed that both the nanocrystalline and coarse-grained samples had transformed fully to the hcp phase at the final pressures measured.

\section{DISCUSSION}

\section{A. High-energy region}

In Fig. 1, a small enhancement is observable in the DOS of the nanocrystalline sample above the high-energy cutoff of the DOS of the bulk sample. This has been adequately explained by the effects of lifetime broadening and the presence of iron oxides. ${ }^{7}$ X-ray-diffraction measurements on the nanocrystalline sample showed the presence of oxide, which is difficult to prevent completely in nanostructured materials owing to their large surface area. Upon the increase of pressure, the high-energy enhancement is decreased, but not eliminated. This behavior is consistent with a constant population of oxide modes and a weakened effect of lifetime en-

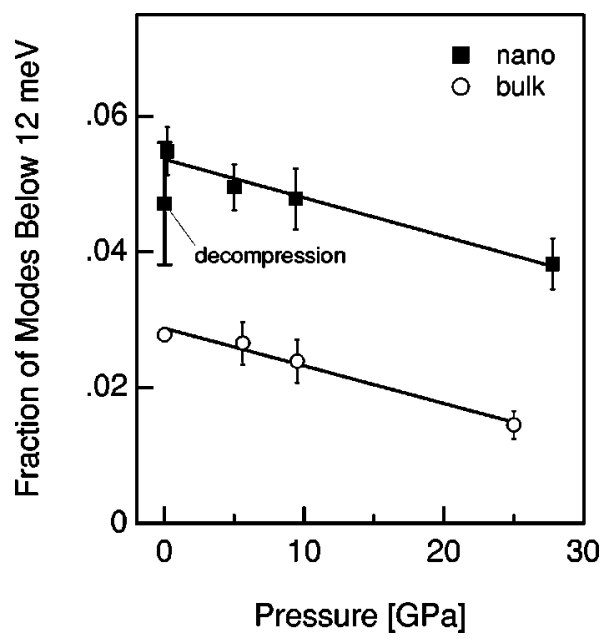

FIG. 3. Fraction of the total number of vibrational modes of energy less than $12 \mathrm{meV}$ in nanocrystalline and bulk ${ }^{57} \mathrm{Fe}$ as a function of pressure. The straight lines are linear fits to the data. ergy broadening as the nanoparticles are compressed. Compression brings individual grains in the nanocrystal into more intimate contact, reducing the strength of elastic discontinuities and decreasing phonon scattering at grain boundaries. The slight sharpening of the longitudinal peak of the nanocrystalline sample at $9 \mathrm{GPa}$ also supports this hypothesis.

Despite the differences observed in the DOS curves, the characteristic van Hove singularities appear at nearly the same energies in both the nanocrystalline and coarse-grained sample, and remain in coincidence at all pressures. As zoneedge phenomena, the singularities are attributes of shortwavelength phonons excited in a regular lattice. The occurrence of these features at the same energies in both microstructures shows that a high grain boundary volume fraction does not distort significantly the vibrational modes inside crystalline regions. The commensurate tracking of the singularities with pressure is evidence that high grain boundary density has little effect on the interatomic potentials for iron atoms within crystalline environments.

\section{B. Low-energy region}

The most obvious difference in the DOS curves of the nanocrystalline and the bulk sample at low energies is the number of modes, as all of the curves are essentially featureless to energies of $\sim 20 \mathrm{meV}$. Figure 3 shows that the fractional enhancement in low-energy modes in nanocrystals remains essentially constant under pressure, and even across the $\alpha \rightarrow \epsilon$ structural phase transition. These results imply that to a large degree, the fraction of grain boundaries, and not their particular structure, is a general source of the enhancement in the low-energy part of the spectrum. This assertion is reinforced by the observation of abundant low-energy modes in nanocrystals with fcc (Ref. 1) and bcc (Ref. 4) structures and those produced by ball milling. ${ }^{3}$

When the nanocrystalline sample was decompressed from its ultimate pressure, Fig. 3 shows that not all of the modes below $12 \mathrm{meV}$ were recovered. This is possibly due to the structural change to the hcp phase at $\sim 12 \mathrm{GPa}$ and back to the bcc phase on decompression. We expect a smaller fraction of the atoms at grain boundaries of a close-packed phase, and this effect may be hysteretic. TEM performed on gas-consolidated nanocrystalline ${ }^{57} \mathrm{Fe}$ that had been compressed to $16 \mathrm{GPa}$ and subsequently decompressed is shown in Fig. 4. The figure shows little change in the shapes and sizes of individual grains, which eliminates the possibility of a lowered fraction of surface area. However, the TEM results do not rule out some densification of grain boundary regions, which have widths on the order of $1 \mathrm{~nm}$.

Seminal work by Van Hove ${ }^{23}$ showed that for low frequencies, the phonon DOS should be proportional to $E^{d-1}$, where $d$ is the dimensionality of the system. The relevant dimensionality remains an unsettled issue for nanostructured materials. It is tempting, but incorrect, to assume that the total DOS can be decomposed into a "grain boundary" partial DOS and a "grain interior" partial DOS. Especially problematic are systems in which the crystallite size approaches the grain boundary width. With a sound velocity of $3500 \mathrm{~m} / \mathrm{sec}$, a $10 \mathrm{meV}$ phonon has a wavelength of $1.5 \mathrm{~nm}$. 

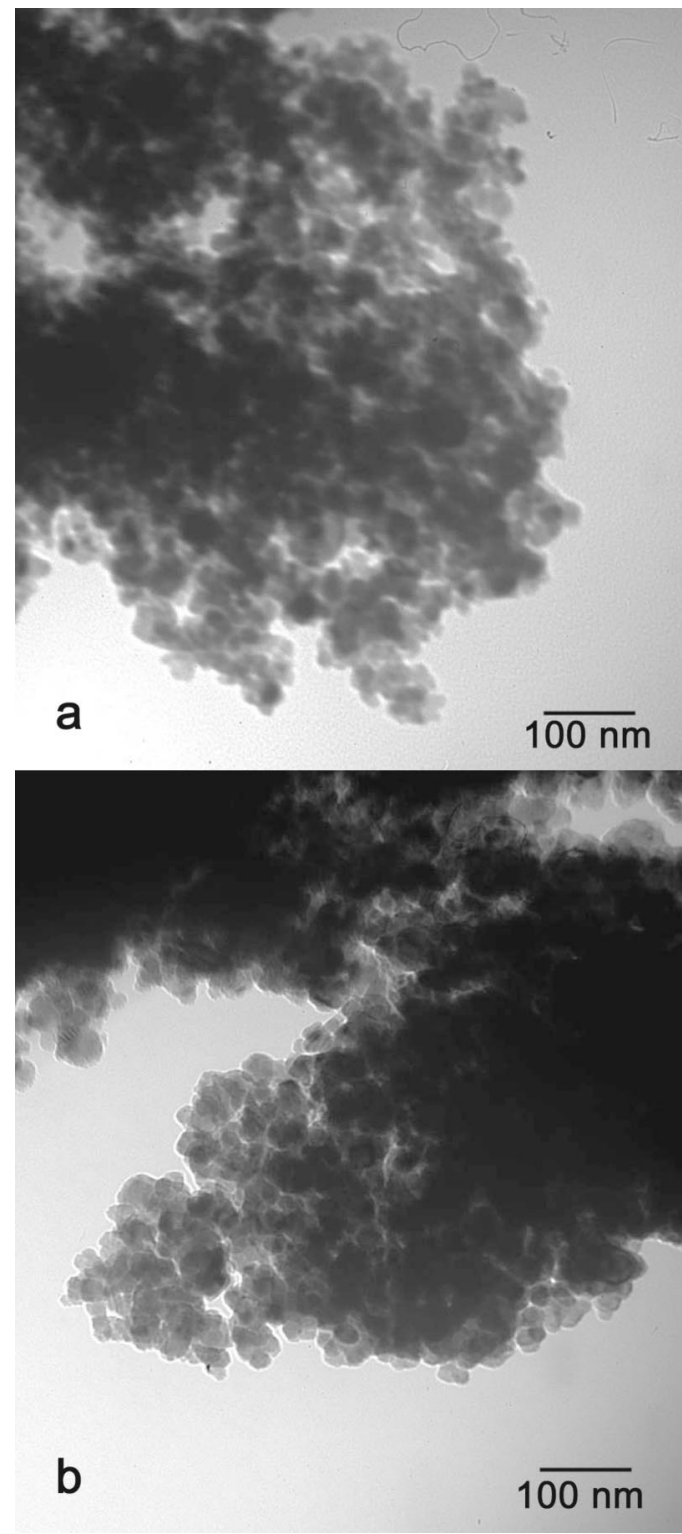

FIG. 4. Bright field TEM image of gas-consolidated ${ }^{57} \mathrm{Fe}$ (a) before and (b) after compression to $16 \mathrm{GPa}$ in a diamond anvil cell.

In a spherical nanocrystal of $9 \mathrm{~nm}$ diameter, $70 \%$ of the atoms in the crystal are within this $1.5 \mathrm{~nm}$ distance of the grain boundary, and this fraction is even larger for crystals with anisotropic shapes. The dynamics of grain boundaries and crystal interiors are therefore coupled closely in a nanocrystalline material. A three-dimensional dynamics may therefore be expected in spite of the large fraction of internal surface in the nanostructure.

The low-energy regions of our measured DOS curves were fit to a power law of the form $A E^{n}$, and the results presented in Fig. 5. Averaging over all data, the value of $n$ is found to be $(2.02 \pm 0.025)$ for the bulk control samples and $(1.98 \pm 0.015)$ for the nanocrystalline material. Both are nearly equal to the value of 2 for three-dimensional vibrational dynamics. The exponent for the data from the nanocrystalline bec $\alpha$ phase alone is $(1.95 \pm 0.015)$, admitting a possible small effect of lower dimensionality. Pressure has

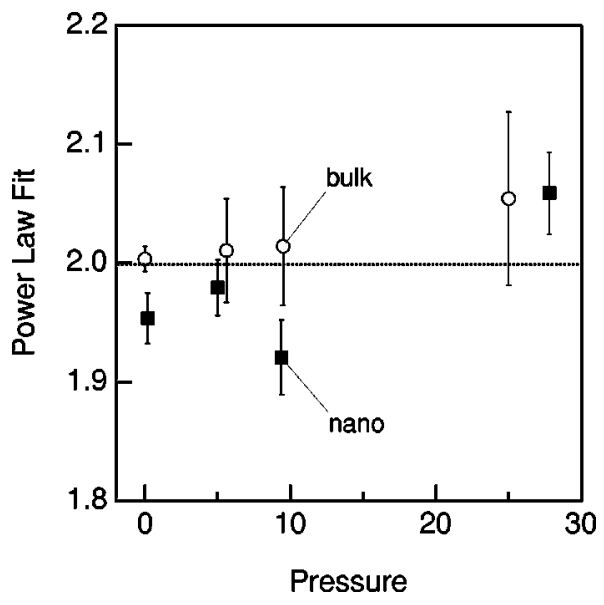

FIG. 5. Fitted exponents $n$ in $A E^{n}$ for the energy dependence of the phonon DOS of nanocrystalline and bulk ${ }^{57} \mathrm{Fe}$ for $E$ below $12 \mathrm{meV}$.

little effect on the exponent $n$, which is not surprising, since $n$ is so close to 2 at ambient pressure. Our results are in good agreement with recent NRIXS and neutron scattering experiments at ambient pressure. ${ }^{4-7}$ All values of $n$ are far removed from the value of 1.5 found by Derlet et al. for the vibrations of grain boundary atoms. We note that another report of experimental evidence for a linear contribution to the spectrum rested on the assumption that the projection on $\mathrm{H}$ atoms of the low-energy modes is the same as the projection on neighboring $\mathrm{Pd}$ atoms. ${ }^{11}$ This is not justified when motions of adjacent Pd atoms are out-of-phase, for example.

\section{CONCLUSION}

Phonon DOS curves of gas-consolidated nanocrystalline iron were measured at pressures up to $28 \mathrm{GPa}$. Compared to bulk polycrystalline iron in the same pressure range, the nanocrystalline sample exhibited a factor of 2.2 more modes in the low-energy region from 0 to $12 \mathrm{meV}$. This enhancement was approximately independent of pressure or even crystal structure. This ratio decreased to 1.7 after decompression, perhaps owing to atom rearrangements within the grain boundaries. The low-energy region of the phonon DOS was fitted to the function $A E^{n}$. Averaging over all data for the nanocrystalline material, $n$ was found to be nearly equal to the value of 2 expected for three-dimensional vibrational dynamics. This rules out major effects from two-dimensional dynamics in the nanostructure. The van Hove singularities of both samples occurred at nearly the same energies and tracked commensurately with pressure. The low-energy modes of the phonon DOS in nano-Fe are best explained as modes of a coupled three-dimensional system. The pressure dependence of these low-energy modes is nearly the same as that of the normal crystalline phonons.

\section{ACKNOWLEDGMENTS}

This work was supported by the U.S. National Science Foundation under Grant No. DMR-0204920. Use of the Advanced Photon Source is supported by the U.S. Department of Energy under Contract No. W-31-109-ENG-38. 
${ }^{1}$ B. Fultz, L. Anthony, L. Nagel, R. Nicklow, and S. Spooner, Phys. Rev. B 52, 3315 (1995).

${ }^{2}$ J. Trampenau, K. Bauszus, W. Petry, and U. Herr, Nanostruct. Mater. 6, 551 (1995).

${ }^{3}$ B. Fultz, J. Robertson, T. Stephens, L. Nagel, and S. Spooner, J. Appl. Phys. 79, 8318 (1996).

${ }^{4}$ B. Fultz, C. Ahn, E. Alp, W. Sturhahn, and T. Toellner, Phys. Rev. Lett. 79, 937 (1997).

${ }^{5}$ H. Frase, B. Fultz, and J. Robertson, Phys. Rev. B 57, 898 (1998).

${ }^{6}$ E. Bonetti, L. Pasquini, E. Sampaolesi, A. Deriu, and G. Cicognani, J. Appl. Phys. 88, 4571 (2000).

${ }^{7}$ L. Pasquini, A. Barla, A. Chumakov, O. Leupold, R. Ruffer, A. Deriu, and E. Bonetti, Phys. Rev. B 66, 073410 (2002).

${ }^{8}$ G. Von Eynatten, J. Horst, K. Dransfeld, and H. Bömmel, Hyperfine Interact. 29, 1311 (1986).

${ }^{9}$ A. Kara and T. Rahman, Phys. Rev. Lett. 81, 1453 (1998).

${ }^{10}$ D. Sun, X. Gong, and X. Wang, Phys. Rev. B 63, 193412 (2001).

${ }^{11}$ U. Stuhr, H. Wipf, K. Andersen, and H. Hahn, Phys. Rev. Lett. 81, 1449 (1998).

${ }^{12}$ P. Derlet, R. Meyer, L. Lewis, U. Stuhr, and H. Van Swygen- hoven, Phys. Rev. Lett. 87, 205501 (2001).

${ }^{13}$ J. Chadwick, J. Phys. A 32, 4087 (1999).

${ }^{14}$ R. Orbach, Science 231, 814 (1986).

${ }^{15}$ W. Sturhahn, T. Toellner, E. Alp, X. Zhang, M.S.S. Kikuta, C. Kimball, and B. Dabrowski, Phys. Rev. Lett. 74, 3832 (1995).

${ }^{16} \mathrm{~J}$. Reid, R. Brain, and C. Ahn, in Proceedings of the 12th International VLSI Multilevel Interconnect Conference, edited by $\mathrm{T}$. Wade (Santa Clara, CA, 1995), p. 545.

${ }^{17} \mathrm{~T}$. Toellner (unpublished).

${ }^{18}$ H. Mao et al., Science 292, 914 (2001).

${ }^{19}$ M. Hu, W. Sturhahn, T. Toellner, P. Hession, J. Sutter, and E. Alp, Nucl. Instrum. Methods Phys. Res. A 428, 551 (1999).

${ }^{20}$ R. Forman, G. Piermarini, J. Barnett, and S. Block, Science 176, 284 (1972).

${ }^{21}$ W. Sturhahn, T. Toellner, E. Alp, P. Hession, M. Hu, and J. Sutter, Hyperfine Interact. 113, 47 (1998).

${ }^{22}$ R. Lübbers, G. Wortmann, and H. Grünsteudel, Hyperfine Interact. 123/124, 529 (1999).

${ }^{23}$ L. Van Hove, Phys. Rev. 89, 1189 (1953). 